OPEN ACCESS

Edited by:

Qin Zhou,

Sun Yat-Sen University, China

Reviewed by:

Xiaoqun Dong,

Brown University, United States

Jing He,

Guangzhou Medical University, China

*Correspondence:

Wenquan Niu

niuwenquan_shcn@163.com; niuwenquan@zryhyy.com.cn

Ping $\mathrm{Li}$

Ip8675@163.com

Yongtong $\mathrm{CaO}$

caoyongtong92@sina.com

Specialty section:

This article was submitted to

Translational Medicine,

a section of the journal

Frontiers in Medicine

Received: 27 January 2021 Accepted: 12 March 2021

Published: 06 April 2021

Citation:

Ma L, Wang S, Zhao H, Yu M, Deng $X$, Jiang Y, Cao Y, Li P and Niu W (2021)

Susceptibility of ApoB and PCSK9

Genetic Polymorphisms to Diabetic Kidney Disease Among Chinese

Diabetic Patients.

Front. Med. 8:659188.

doi: 10.3389/fmed.2021.659188

\section{Susceptibility of $A p o B$ and PCSK9 Genetic Polymorphisms to Diabetic Kidney Disease Among Chinese Diabetic Patients}

\author{
Liang $\mathrm{Ma}^{1}$, Shaoting Wang ${ }^{1}$, Hailing Zhao ${ }^{2}$, Meijie $\mathrm{Yu}^{3}$, Xiangling Deng ${ }^{4}$, Yongwei Jiang ${ }^{1}$, \\ Yongtong $\mathrm{CaO}^{1 *}$, Ping $\mathrm{Li}^{2 *}$ and Wenquan $\mathrm{Niu}^{4 *}$ \\ ${ }^{1}$ Clinical Laboratory, China-Japan Friendship Hospital, Beijing, China, ${ }^{2}$ Beijing Key Laboratory of Immune-Mediated \\ Inflammatory Diseases, Institute of Clinical Medical Science, China-Japan Friendship Hospital, Beijing, China, ${ }^{3}$ Department of \\ Nephrology, China-Japan Friendship Hospital, Beijing, China, ${ }^{4}$ Institute of Clinical Medical Science, China-Japan Friendship \\ Hospital, Beijing, China
}

This study aimed to investigate the susceptibility of 8 polymorphisms in $A p o B$ and PCSK9 genes to diabetic kidney disease (DKD) in Chinese patients with type 2 diabetes mellitus. This is a case-control association study, including 575 DKD cases and 653 controls. Genotypes were determined using ligase detection reaction method, and data are analyzed using STATA software. The genotype distributions of rs1042034 and rs12720838 differed significantly between the two groups $(P<0.001$ and $P=0.008$, respectively). After adjusting for confounding factors, the mutations of rs1042034 and rs12720838 were associated with the significantly increased risk of DKD. For instance, carriers of rs1042034 T allele (CT and TT genotypes) were 1.07 times more likely to have DKD than carriers of rs $1042034 \mathrm{CC}$ genotype [odds ratio $(\mathrm{OR})=1.07,95 \%$ confidence interval (Cl): 1.03-1.10, $P<0.001]$. Further, haplotype T-A-G-T in $A p o B$ gene was overrepresented in cases (18.10\%) compared with controls (12.76\%) (Psimulated $=0.045)$, and haplotype T-A-G-T was associated with a 33\% increased risk of DKD $(\mathrm{OR}=1.33$, $95 \% \mathrm{Cl}: 1.04,1.70)$. In further haplotype-phenotype analysis, significant association was only noted for hypertension and omnibus haplotypes in $A p o B$ gene ( $\left.P_{\text {simulated }}=0.001\right)$. Our findings indicate that $A p o B$ gene is a candidate gene for DKD in Chinese patients with type 2 diabetes mellitus.

Keywords: diabetic kidney disease, single nucleotide polymorphism, association, ApoB/PCSK9 genes, risk

\section{INTRODUCTION}

As a major microvascular complication of diabetes mellitus, diabetic kidney disease (DKD) has skyrocketed to epidemic proportions. Latest statistics indicates that the age-standardized prevalence of DKD worldwide was 15.48/1,000 and 16.50/1,000 in men and women, respectively (1). Although global DKD prevalence has remained stabilized during the last three decades, the mortality rate of DKD is growing in comparison to that of other types of chronic kidney disease (2). Effective strategies should be developed to curb this global burden (3). A practical strategy is the identification of possible risk factors to potentially predict subjects who are more likely to pre-dispose to $\mathrm{DKD}$, thereby helping doctors and healthcare professionals to make immediate prevention and control measures against this disease. 
It is widely accepted that DKD is a complex multifactorial disease, partly under genetic control (4). Previous evidence revealed that DKD occurs in familial clusters, indicating a strong heritable component in the pathogenesis $(5,6)$. The results of recently completed genome-wide association studies have advanced knowledge on the genetic underpinnings of DKD (7-10). Despite much endeavors, the causal genetic determinants of DKD are not yet fully understood. As such, candidate gene approach represents an alternative strategy by focusing on the genes with strong biological or clinical implications (11). The genes encoding apolipoprotein B (ApoB) and proprotein convertase subtilisin/kexin type 9 (PCSK9) are such candidate genes for DKD. Clinical studies have shown that circulating ApoB served as an independent predictor of renal replacement therapy in DKD patients $(12,13)$, and PCSK9 can promote hypercholesterolemia, and PCSK9 concentrations were associated with chronic kidney disease stages (14) and lipidlowering regimens (15). However, in the medical literature, the genetic pre-disposition of $A p o B$ and PCSK9 genes to DKD is rarely reported.

To fill this gap in knowledge and yield more information for future research, we genotyped 5 single nucleotide polymorphisms (SNPs) in $A p o B$ gene and 3 SNPs in PCSK9 gene in 575 DKD patients and 653 controls, and investigated their susceptibility to DKD risk, both individually and jointly.

\section{METHODS}

\section{Study Design and Ethical Approval}

This is a case-control association study officially approved by the institutional review boards of the China-Japan Friendship Hospital. All study subjects were enrolled from this hospital during the period between August 2016 and December 2018, and they gave written informed consent prior to drawing blood samples for genetic analysis and filling out questionnaires.

\section{Study Subjects}

A total of 1,228 patients, who were diagnosed with type 2 diabetes mellitus, were enrolled in this study. Of these patients, 575 had newly-diagnosed and histologically-confirmed DKD, as the case group. The remaining 653 patients who had experienced type 2 diabetes mellitus for 7 or more years, were clinically confirmed to be free of DKD, and had no history of severe kidney diseases formed the control group.

\section{Eligibility Criteria}

DKD was diagnosed according to the National Kidney Foundation Kidney Disease Outcomes Quality Initiative (NKF-K/DOQI) guidelines (16).

In detail, subjects in the case group were included if they had a clinical diagnosis of type 2 diabetes mellitus and $24 \mathrm{~h}$ urinary albumin $>500 \mathrm{mg} / 24 \mathrm{~h}$ or an albumin creatinine ratio $(\mathrm{ACR})>30 \mathrm{mg} / \mathrm{g}$, and subjects were excluded if they had no previous history of kidney diseases, or if they had primary or secondary kidney diseases that caused proteinuria, such as IgA nephropathy, membranous nephropathy, lupus nephritis, obstructive renal disease, and acute urinary tract infection.
Subjects in the control group were included if they had a clinical diagnosis of type 2 diabetes mellitus and ACR $<30 \mathrm{mg} / \mathrm{g}$. Exclusion criteria were identical as the case group.

\section{Demographic Data Collection}

A structured questionnaire was designed to collect information on age, gender, body weight/height, cigarette smoking, hypertension status, and duration of type 2 diabetes mellitus. Body mass index (BMI) was calculated as body weight (in kilometers) divided by body height (in meters) squared.

\section{Laboratory Biomarker Measurement}

Laboratory markers included $24 \mathrm{~h}$ urinary albumin excretion, ACR, high density lipoprotein cholesterol (HDLC), low density lipoprotein cholesterol (LDLC), total cholesterol (TC), triglyceride (TG), hemoglobin A1c (HbAlc), and homocysteine (HCY). Serum concentrations of fasting TG, TC, HDLC, LDLC, and HCY were measured using an automated biochemical analyzer (AU5800 Clinical Chemistry System, Beckman Coulter, Brea, CA, USA). HBA1c was measured using the D-10 Hemoglobin Testing System (Bio-Rad, Hercules, CA, USA).

\section{Genomic DNA Extraction and Genotyping}

Genomic DNA was extracted from whole blood cells according to the manufacturer instructions, and quantified using the NanoDrop 1000 spectrophotometer (ThermoScientific). DNA samples were frozen at $-20^{\circ} \mathrm{C}$ until mass genotyping.

Five SNPs in $A p o B$ gene, including rs1042034, rs679899, rs676210, rs1367117, rs12720838, and three SNPs in PCSK9 gene, including rs662145, rs45448095, rs11583680, were genotyped by use of the ligase detection reaction (LDR) method.

In detail, 50 ng DNA was amplified in $15 \mu \mathrm{l}$ reaction mixture containing $7.5 \mu \mathrm{L}$ of Premix Ex Taq, and $10 \mathrm{pmol}$ of each primer for the amplification of genomic sequences. Pre-heating of the mixture at $94^{\circ} \mathrm{C}$ for 3 min followed by 35 cycles of denaturation at $94^{\circ} \mathrm{C}$ for $15 \mathrm{~s}$, annealing at $55^{\circ} \mathrm{C}$ for $30 \mathrm{~s}$, and elongation at $75^{\circ} \mathrm{C}$ for $90 \mathrm{~s}$. LDR ligation reaction was in $10 \mu \mathrm{l}$ reaction mixture containing $3 \mu \mathrm{l}$ of polymerase chain reaction (PCR) product, 1 $\mu l$ of $10^{*}$ Taq DNA ligase buffer, $0.125 \mu l$ of Taq DNA ligase, and $0.02 \mu \mathrm{l}$ of mix probes.

The primer sequences and probe sequences of each SNP are summarized in Supplementary Tables 1, 2, respectively.

\section{Statistical Analysis}

Continuous data are expressed as median (interquartile range), and categorical data as percentage. Two-group comparisons were completed using the Wilcoxon rank-sum test or $\chi^{2}$-test when appropriate. The genotypes and alleles of each SNP under study, as well as the tests for Hardy-Weinberg equilibrium, were compared using $\chi^{2}$-test between controls and cases. Risk prediction of each SNP for DKD, summarized as odds ratio (OR) and 95\% confidence interval (CI), was calculated separately under additive and dominant models before and after adjusting for confounding factors. Linkage patterns of SNPs under study in each gene were examined using the HaploView software (version 4.2, Cambridge, MA, USA). 
The frequencies of derived haplotypes were estimated using the Haplo.Stats program in the $\mathrm{R}$ software (version 3.6.1). Comparison of derived haplotypes between the two groups, prediction estimates of each haplotype for DKD risk, and association of omnibus haplotypes with baseline characteristics were completed using the Haplo.Stats program as well.

Unless otherwise stated, statistical analyses were performed using the STATA software (version 14.1, Stata Corp., College Station, TX). The power to detect statistical significance was derived using the PS Power and Sample Size Calculations (version 3.0) (17).

\section{RESULTS}

\section{Baseline Characteristics}

Table 1 shows the baseline characteristics of study subjects. Cases were significantly older than controls (median age, 62 vs. 60 years, $P=0.001)$. There was a slightly high proportion of males in cases relative to controls $(66.4 \%$ vs. $60.8, P=0.041)$, as well as a high proportion of smokers $(P=0.041)$ and hypertension $(P<0.001)$.

\section{Linkage Disequilibrium}

In $A p o B$ gene, two SNPs, rs1042034 and rs676210, were in complete linkage, and in PCSK9 gene, rs45448095 and rs11583680 were in complete linkage. As such, rs676210 and rs11583680 were removed from the following analyses.

\section{Genotype and Allele Distributions}

The genotype and allele distributions of the remaining 6 SNPs between controls and cases are presented in Table 2. The genotype distributions of rs1042034 and rs12720838 differed significantly between the two groups $(P<0.001$ and $P=0.008$,

\begin{tabular}{lccr}
\hline \multicolumn{4}{c}{ TABLE 1 | The baseline characteristics of the study subjects. } \\
\hline Characteristics & $\begin{array}{c}\text { Controls } \\
(\boldsymbol{n}=\mathbf{6 5 3})\end{array}$ & $\begin{array}{c}\text { Cases } \\
(\boldsymbol{n}=\mathbf{5 7 5})\end{array}$ & $\boldsymbol{P}$ \\
\hline Age, years & $60(53,67)$ & $62(54,70)$ & 0.001 \\
Males, \% & 60.8 & 66.4 & 0.041 \\
Smokers, \% & 32.0 & 37.6 & 0.041 \\
Hypertension, \% & 51.6 & 78.1 & $<0.001$ \\
BMI, kg/m & $25.31(23.40,27.70)$ & $25.78(24.00,28.39)$ & 0.003 \\
Duration of & $14(10,18)$ & $15(9,21)$ & 0.208 \\
diabetes, years & & & \\
TC, mmol/L & $4.15(3.50,4.88)$ & $4.19(3.43,5.03)$ & 0.715 \\
HbA1C, mmol/L & $7.85(6.80,9.20)$ & $7.80(6.70,9.30)$ & 0.984 \\
HCY, $\mu \mathrm{mol} / \mathrm{L}$ & $11.10(9.41,13.26)$ & $13.37(10.74,16.81)$ & $<0.001$ \\
TG, mmol/L & $1.45(1.03,2.18)$ & $1.65(1.13,2.44)$ & $<0.001$ \\
HDLC, mmol/L & $0.99(0.83,1.20)$ & $0.97(0.79,1.19)$ & 1.578 \\
LDLC, mmol/L & $2.44(1.93,3.06)$ & $2.39(1.83,3.08)$ & 1.768 \\
\hline BM & & &
\end{tabular}

$B M I$, body mass index; SBP, systolic blood pressure; $D B P$, diastolic blood pressure; TC, total cholesterol; HbA1C, hemoglobin A1C; HCY, homocysteine; TG, triglyceride; HDLC, high-density lipoprotein cholesterol; LDLC, low-density lipoprotein cholesterol. Data are expressed as median (interquartile range) or percentage, when appropriate. $P$ was calculated using the Wilcoxon rank-sum test for continuous data and the $\chi^{2}$-test for categorical data. respectively). No significance was noted for the comparisons of the other SNPs. The genotype distributions of all study SNPs satisfied the Hardy-Weinberg equilibrium at a significance level of $10 \%$.

\section{Single-Locus Analysis}

The risk prediction of the remaining 6 SNPs for DKD risk under both additive and dominant models is provided in Table 3. After adjusting for confounding factors, including age, gender, smoking, BMI, hypertension, and duration of diabetes, the mutations of rs1042034 and rs12720838 were associated with the significantly increased risk of DKD under both models of inheritance. For instance, carriers of rs1042034 T allele (CT and TT genotypes) were 1.07 times more likely to have DKD than carriers of rs1042034 CC genotype, independent of these confounders $(\mathrm{OR}=1.07,95 \% \mathrm{CI}: 1.03-1.10, P<0.001)$.

\section{Haplotype Analysis}

Table 4 shows the estimated frequencies of haplotypes separately in $A p o B$ and PCSK9 genes, as well as their prediction for DKD

TABLE 2 | The genotype and allele distributions of studied polymorphisms between controls and cases.

\begin{tabular}{llllll}
\hline SNPs & $\begin{array}{l}\text { Genotype/ } \\
\text { allele }\end{array}$ & Controls & Cases & $\chi^{2}$ & $P^{*}$ \\
\hline
\end{tabular}

\begin{tabular}{|c|c|c|c|c|c|}
\hline \multicolumn{6}{|l|}{$A p o B$ gene } \\
\hline \multirow[t]{4}{*}{ rs1042034 } & $\mathrm{CC}$ & 373 (57.12\%) & 319 (55.48\%) & 18.11 & $<0.001$ \\
\hline & CT & $270(41.35 \%)$ & $221(38.43 \%)$ & & \\
\hline & $\pi$ & 10 (1.53\%) & 35 (6.09\%) & & \\
\hline & $\mathrm{T}$ & $290(22.21 \%)$ & 291 (25.30\%) & 3.25 & 0.071 \\
\hline \multirow[t]{4}{*}{ rs679899 } & $\mathrm{AA}$ & 453 (69.37\%) & 409 (71.13\%) & 3.27 & 0.195 \\
\hline & $A G$ & $188(28.79 \%)$ & $148(25.74 \%)$ & & \\
\hline & GG & $12(1.84 \%)$ & 18 (3.13\%) & & \\
\hline & G & 212 (16.23\%) & $172(15.11 \%)$ & 0.57 & 0.448 \\
\hline \multirow[t]{4}{*}{ rs1367117 } & GG & 496 (75.96\%) & 434 (75.48\%) & 0.04 & 0.981 \\
\hline & $A G$ & $147(22.51 \%)$ & $132(22.96 \%)$ & & \\
\hline & $\mathrm{AA}$ & 10 (1.53\%) & $9(1.57 \%)$ & & \\
\hline & A & 167 (12.79\%) & 150 (13.04\%) & 0.04 & 0.850 \\
\hline \multirow[t]{4}{*}{ rs12720838 } & $\mathrm{CC}$ & 455 (69.68\%) & 393 (68.35\%) & 9.70 & 0.008 \\
\hline & CT & 192 (29.4\%) & 162 (28.17\%) & & \\
\hline & $\pi$ & $6(0.92 \%)$ & 20 (3.48\%) & & \\
\hline & $\mathrm{T}$ & 204 (15.62\%) & 202 (17.57\%) & 1.68 & 0.195 \\
\hline \multicolumn{6}{|l|}{ PCSK9 gene } \\
\hline \multirow[t]{4}{*}{ rs662145 } & $\pi$ & 505 (77.34\%) & 434 (75.48\%) & 1.34 & 0.511 \\
\hline & CT & 137 (20.98\%) & 134 (23.30\%) & & \\
\hline & $\mathrm{CC}$ & 11 (1.68\%) & 7 (1.22\%) & & \\
\hline & C & 159 (12.17\%) & 148 (12.87\%) & 0.27 & 0.603 \\
\hline \multirow[t]{4}{*}{ rs45448095 } & $\mathrm{CC}$ & 544 (83.31\%) & 454 (78.96\%) & 3.84 & 0.147 \\
\hline & CT & 103 (15.77\%) & 115 (20.00\%) & & \\
\hline & $\pi$ & $6(0.92 \%)$ & $6(1.04 \%)$ & & \\
\hline & $\mathrm{T}$ & 115 (8.81\%) & 127 (11.04\%) & 3.45 & 0.063 \\
\hline
\end{tabular}

SNPs, single nucleotide polymorphisms. ${ }^{*} P$ was calculated using the $\chi^{2}$-test. 
risk. In $A p o B$ gene, the most common haplotype was C-AG-C (alleles in order of rs1042034, rs679899, rs1367117, and rs12720838), and its frequency was $66.54 \%$ in controls and $60.90 \%$ in cases. In PCSK9 gene, the most common haplotype was T-C (alleles in order of rs662145 and rs45448095), and its frequency was $80.19 \%$ in controls and $82.28 \%$ in cases.

TABLE 3 | Risk prediction of 8 studied polymorphisms for diabetic kidney disease under both additive and dominant models.

\begin{tabular}{|c|c|c|c|c|}
\hline \multirow[t]{2}{*}{ SNPs } & \multicolumn{2}{|c|}{ Additive model } & \multicolumn{2}{|c|}{ Dominant model } \\
\hline & OR (95\% Cl) & $P$ & OR (95\% Cl) & $P$ \\
\hline \multicolumn{5}{|c|}{ Before adjustment } \\
\hline rs1042034 & $1.06(1.03,1.10)$ & $<0.001$ & $1.06(1.03,1.10)$ & $<0.001$ \\
\hline rs679899 & $1.02(0.99,1.05)$ & 0.257 & $1.02(0.99,1.05)$ & 0.257 \\
\hline rs1367117 & $1.00(0.99,1.01)$ & 0.845 & $1.00(0.99,1.01)$ & 0.845 \\
\hline rs12720838 & $1.06(1.02,1.10)$ & 0.005 & $1.06(1.02,1.1)$ & 0.005 \\
\hline rs662145 & $1.00(0.98,1.01)$ & 0.459 & $1.00(0.98,1.01)$ & 0.459 \\
\hline rs45448095 & $1.02(0.97,1.08)$ & 0.391 & $1.02(0.97,1.08)$ & 0.391 \\
\hline \multicolumn{5}{|c|}{ After adjustment* } \\
\hline rs1042034 & $1.07(1.03,1.10)$ & $<0.001$ & $1.07(1.03,1.10)$ & $<0.001$ \\
\hline rs679899 & $1.02(0.99,1.05)$ & 0.287 & $1.02(0.99,1.05)$ & 0.287 \\
\hline rs1367117 & $1.00(0.99,1.01)$ & 0.883 & $1.00(0.99,1.01)$ & 0.883 \\
\hline rs12720838 & $1.06(1.02,1.11)$ & 0.002 & $1.06(1.02,1.11)$ & 0.002 \\
\hline rs662145 & $0.99(0.98,1.01)$ & 0.334 & $0.99(0.98,1.01)$ & 0.334 \\
\hline rs45448095 & $1.02(0.97,1.07)$ & 0.506 & $1.02(0.97,1.07)$ & 0.506 \\
\hline
\end{tabular}

SNPs, single nucleotide polymorphisms; OR, odds ratio; $95 \% \mathrm{Cl}$, 95\% confidence interval. *Adjusting for age, gender, smoking, body mass index, hypertension, and duration of diabetes.
Comparison of haplotypes between the two groups revealed that haplotype T-A-G-T in $A p o B$ gene was overrepresented in cases (18.10\%) compared with controls (12.76\%) (Simulated $P=0.045)$, and haplotype T-A-G-T was associated with a $33 \%$ increased risk of $\mathrm{DKD}(\mathrm{OR}=1.33,95 \% \mathrm{CI}: 1.04,1.70)$. The power to detect the significant association of haplotype T-A-G-T with DKD was estimated to be $79.9 \%$.

No significance was noted for the comparisons of other haplotypes and risk predictions.

\section{Haplotype-Phenotype Analysis}

Further, an analysis on the association of haplotypes as a whole with baseline characteristics was done in both genes (Table 5). Of all characteristics, significant association was only noted for hypertension and omnibus haplotypes in $A p o B$ gene (Simulated $P=0.001)$.

\section{DISCUSSION}

To the best of our knowledge, this is the first study that has explored the susceptibility of $A p o B$ and PCSK9 genetic alternations to $\mathrm{DKD}$ risk in a large Chinese diabetic population. The key finding of this study is that $A p o B$ gene is a candidate gene for DKD. Particularly, two SNPs, rs1042034 and rs12720838, in $A p o B$ gene were individually associated with the significant risk of DKD under both additive and dominant models of inheritance, and in the presence of other two SNPs in this gene, the risk was clearly enhanced, indicating the possible synergistic contribution.

DKD is a polygenic disease (18). A long list of genes have been identified to play a contributory role in the pathogenesis of

TABLE 4 | The frequency of derived haplotypes between controls and cases, and their risk prediction for diabetic kidney disease.

\begin{tabular}{|c|c|c|c|c|c|c|}
\hline Haplotype* & Controls (\%) & Cases (\%) & Hap. score & $\boldsymbol{P}$ & Simulated $\boldsymbol{P}$ & OR (95\% Cl) \\
\hline \multicolumn{7}{|l|}{$A p o B$ gene } \\
\hline C-A-G-C & 66.54 & 60.90 & -1.39 & 0.163 & 0.175 & Reference \\
\hline T-A-G-T & 12.76 & 18.10 & 1.81 & 0.042 & 0.045 & $1.33(1.04,1.70)$ \\
\hline C-G-A-C & 6.28 & 4.64 & -0.83 & 0.408 & 0.410 & $0.94(0.66,1.35)$ \\
\hline T-G-A-C & 4.35 & 4.92 & 0.68 & 0.497 & 0.530 & $1.22(0.82,1.81)$ \\
\hline T-G-G-C & 2.99 & 3.23 & 0.32 & 0.749 & 0.700 & $1.14(0.70,1.87)$ \\
\hline C-G-G-C & 1.84 & 2.21 & -0.10 & 0.924 & 0.925 & $1.22(0.64,2.35)$ \\
\hline C-A-A-C & 1.34 & 2.49 & 1.24 & 0.213 & 0.210 & $1.94(0.97,3.89)$ \\
\hline C-A-G-T & 1.64 & 1.46 & -0.62 & 0.537 & 0.555 & $0.94(0.47,1.89)$ \\
\hline T-A-G-C & 0.98 & 1.05 & 0.13 & 0.900 & 0.920 & $1.15(0.49,2.69)$ \\
\hline \multicolumn{7}{|l|}{ PCSK9 gene } \\
\hline $\mathrm{T}-\mathrm{C}$ & 80.19 & 82.28 & -1.34712 & 0.178 & 0.185 & Reference \\
\hline $\mathrm{C}-\mathrm{C}$ & 8.77 & 8.91 & -0.13257 & 0.895 & 0.885 & $1.01(0.75,1.35)$ \\
\hline $\mathrm{T}-\mathrm{T}$ & 6.94 & 5.54 & 1.47107 & 0.141 & 0.105 & $1.28(0.91,1.8)$ \\
\hline C-T & 4.10 & 3.26 & 1.20819 & 0.227 & 0.210 & $1.3(0.82,2.06)$ \\
\hline
\end{tabular}

OR, odds ratio; 95\% Cl, 95\% confidence interval.

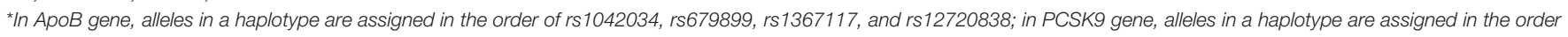
of rs662145 and rs45448095. 
TABLE 5 | Association of all derived haplotypes as a whole in each gene with baseline characteristics of study subjects.

\begin{tabular}{|c|c|c|c|c|c|c|}
\hline \multirow[t]{2}{*}{ Characteristics } & \multicolumn{3}{|c|}{ ApoB gene } & \multicolumn{3}{|c|}{ PCSK9 gene } \\
\hline & Global statistics & $P$ & Simulated $P$ & Global statistics & $P$ & Simulated $P$ \\
\hline Age & 13.08 & 0.442 & 0.269 & 2.57 & 0.462 & 0.451 \\
\hline Gender & 24.27 & 0.029 & 0.069 & 6.00 & 0.112 & 0.104 \\
\hline BMl & 8.93 & 0.778 & 0.497 & 1.46 & 0.692 & 0.698 \\
\hline Duration of diabetes & 11.72 & 0.468 & 0.307 & 1.28 & 0.734 & 0.759 \\
\hline Hypertension & 104.82 & $<0.001$ & 0.001 & 1.12 & 0.773 & 0.794 \\
\hline Smoking & 13.81 & 0.387 & 0.283 & 0.78 & 0.854 & 0.862 \\
\hline TC & 12.14 & 0.516 & 0.257 & 1.37 & 0.712 & 0.709 \\
\hline $\mathrm{HbA} 1 \mathrm{C}$ & 17.48 & 0.178 & 0.101 & 0.29 & 0.963 & 0.962 \\
\hline $\mathrm{HCY}$ & 18.18 & 0.151 & 0.057 & 1.61 & 0.656 & 0.651 \\
\hline TG & 9.51 & 0.733 & 0.371 & 0.44 & 0.933 & 0.950 \\
\hline HDLC & 6.61 & 0.921 & 0.656 & 2.43 & 0.488 & 0.507 \\
\hline LDLC & 13.25 & 0.429 & 0.210 & 2.69 & 0.443 & 0.407 \\
\hline
\end{tabular}

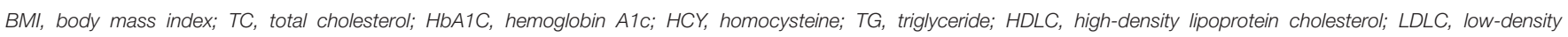
lipoprotein cholesterol.

DKD, such as ADIPOQ gene (19) and IL-6 gene (20). However, the results are not often reproducible at a population level. The reasons behind this poor reproducibility are manifold. The most possible reason is the divergence in the genetic underpinnings of different origins of populations. For instance, a polymorphism may be in close linkage with another nearby causal locus in one ethnic group but not in another (21). As such, it is necessary to establish a candidate list of culprit genes and mutations in each ethnic group. Another reason lies in the fact that the net impact of a single gene or single mutation on complex diseases such as DKD may be small or moderate, or its impact may be offset or antagonized by other cellular regulators (22). To shed some light on this issue, besides single-locus analysis, we also interrogated the contribution of unlinked SNPs as a haplotype, and importantly, the risk magnitude in $A p o B$ gene was reinforced in haplotype analysis. In particular, the significant haplotype T-AG-T in $A p o B$ gene, harboring the mutant alleles of both rs 1042034 and rs12720838, was associated with over 30\% increased risk of $\mathrm{DKD}$, in comparison with the increased risk of both SNPs at 6 and $6 \%$, respectively.

The third possible reason is the involvement of environmental or intermediate phenotypes in the development of DKD, such as hypertension, which has been established as a promising risk factor for $\operatorname{DKD}(23,24)$. As expected, we have observed a significant association between omnibus haplotypes in $A p o B$ gene and hypertension. Dozens of studies have evaluated the genetic susceptibility of $A p o B$ gene to hypertension (25-27). In view of our haplotype-disease and haplotype-phenotype analyses, it is reasonable to speculate that the association of $A p o B$ gene with DKD may be mediated by its association with hypertension, which further precipitates the development of DKD. We agree that further studies exploring the concurrent association of $A p o B$ genetic defects with hypertension and DKD are needed to confirm or refute this speculation.
Several limitations should be acknowledged for this association study. First, this study is cross-sectional and hospital-based in design, and all study subjects were diagnosed to have type 2 diabetes mellitus. It is of added interest to enroll healthy subjects free of type 2 diabetes mellitus as the controls. Second, only 8 candidate SNPs were selected in $A p o B$ and PCSK9 genes. Third, the sample size is insufficient for further subsidiary investigations, such as upon stratification by gender and hypertension. Fourth, all study subjects are exclusively Chinese, and it leaves an open question for the generalizability of our findings to other ethnic groups.

\section{Conclusions}

Despite these limitations, our findings indicate that $A p o B$ gene is a candidate gene for $\mathrm{DKD}$ in Chinese patients with type 2 diabetes mellitus. Although no hint of association was detected for PCSK9 gene, its candidacy in the development of DKD cannot be excluded, and is subject to a matter of debate. For practical reasons, further studies are needed to investigate the underlying mechanisms of ApoB, in vitro or in vivo, in the pathogenesis of DKD.

\section{DATA AVAILABILITY STATEMENT}

The original contributions generated in the study are included in the article/Supplementary Materials, further inquiries can be directed to the corresponding authors.

\section{ETHICS STATEMENT}

The studies involving human participants were reviewed and approved by Institutional Review Boards of the China-Japan 
Friendship Hospital. The patients/participants provided their written informed consent to participate in this study.

\section{AUTHOR CONTRIBUTIONS}

LM, YC, PL, and WN: conceptualization. LM, SW, WN, HZ, XD, YJ, and MY: data collection. LM: funding acquisition. LM, SW, and YJ: investigation. LM, HZ, and XD: data detection. WN: statistics. WN, LM, YC, and PL: writing. All authors read and approved the final manuscript prior to submission.

\section{REFERENCES}

1. Collaborators GBDRF. Global, regional, and national comparative risk assessment of 84 behavioural, environmental and occupational, and metabolic risks or clusters of risks for 195 countries and territories, 1990-2017: a systematic analysis for the Global Burden of Disease Study 2017. Lancet. (2018) 392:1923-94. doi: 10.1016/S0140-6736(18)32225-6

2. Thomas B. The global burden of diabetic kidney disease: time trends and gender gaps. Curr Diab Rep. (2019) 19:18. doi: 10.1007/s11892-019-1133-6

3. Batu Demir D, Cooper ME. New strategies to tackle diabetic kidney disease. Curr Opin Nephrol Hypertens. (2016) 25:348-54. doi: 10.1097/MNH.0000000000000234

4. Florez JC. Genetics of diabetic kidney disease. Semin Nephrol. (2016) 36:474-80. doi: 10.1016/j.semnephrol.2016.09.012

5. Seaquist ER, Goetz FC, Rich S, Barbosa J. Familial clustering of diabetic kidney disease. Evidence for genetic susceptibility to diabetic nephropathy. $N$ Engl J Med. (1989) 320:1161-5. doi: 10.1056/NEJM198905043201801

6. Vijay V, Snehalatha C, Shina K, Lalitha S, Ramachandran A. Familial aggregation of diabetic kidney disease in Type 2 diabetes in south India. Diabetes Res Clin Pract. (1999) 43:167-71. doi: 10.1016/s0168-8227(99)00007-8

7. Palmer ND, Ng MC, Hicks PJ, Mudgal P, Langefeld CD, Freedman BI, et al. Evaluation of candidate nephropathy susceptibility genes in a genome-wide association study of African American diabetic kidney disease. PLOS ONE. (2014) 9:e88273. doi: 10.1371/journal.pone.0088273

8. Iyengar SK, Sedor JR, Freedman BI, Kao WH, Kretzler M, Keller $\mathrm{BJ}$, et al. Genome-wide association and trans-ethnic meta-analysis for advanced diabetic kidney disease: family investigation of nephropathy and diabetes (FIND). PLoS Genet. (2015) 11:e1005352. doi: 10.1371/journal.pgen. 1005352

9. van Zuydam NR, Ahlqvist E, Sandholm N, Deshmukh H, Rayner NW, Abdalla $\mathrm{M}$, et al. A genome-wide association study of diabetic kidney disease in subjects with type 2 diabetes. Diabetes. (2018) 67:1414-27. doi: $10.2337 / \mathrm{db} 17-0914$

10. Salem RM, Todd JN, Sandholm N, Cole JB, Chen WM, Andrews D, et al. Genome-wide association study of diabetic kidney disease highlights biology involved in glomerular basement membrane collagen. J Am Soc Nephrol. (2019) 30:2000-16. doi: 10.1681/ASN.2019030218

11. Kwon JM, Goate AM. The candidate gene approach. Alcohol Res Health. (2000) 24:164-8.

12. Zhao WB, Alberto PSM. Serum apolipoprotein B/apolipoprotein A1 ratio is associated with the progression of diabetic kidney disease to renal replacement therapy. Int Urol Nephrol. (2020) 52:1923-8. doi: 10.1007/s11255-02002550-7

13. Zhao WB, Zhu L, Rahman $\mathrm{T}$. Increased serum concentration of apolipoprotein B is associated with an increased risk of reaching renal replacement therapy in patients with diabetic kidney disease. Ren Fail. (2020) 42:323-8. doi: 10.1080/0886022X.2020.1745235

14. Bermudez-Lopez M, Forne C, Amigo N, Bozic M, Arroyo D, Bretones T, et al. An in-depth analysis shows a hidden atherogenic lipoprotein profile in nondiabetic chronic kidney disease patients. Expert Opin Ther Targets. (2019) 23:619-30. doi: 10.1080/14728222.2019.1620206

\section{FUNDING}

This study was supported by the National Natural Science Foundation of China (Grant Nos: 82074221 and 81703892).

\section{SUPPLEMENTARY MATERIAL}

The Supplementary Material for this article can be found online at: https://www.frontiersin.org/articles/10.3389/fmed. 2021.659188/full\#supplementary-material

15. Elewa U, Fernandez-Fernandez B, Mahillo-Fernandez I, Martin-Cleary C, Sanz AB, Sanchez-Nino MD, et al. PCSK9 in diabetic kidney disease. Eur J Clin Invest. (2016) 46:779-86. doi: 10.1111/eci.12661

16. Kdoqi. KDOQI clinical practice guidelines and clinical practice recommendations for diabetes and chronic kidney disease. Am J Kidney Dis. (2007) 49(Suppl. 2):S12-154. doi: 10.1053/j.ajkd.2006.12.005

17. Dupont WD, Plummer WD, Jr. Power and sample size calculations. A review and computer program. Control Clin Trials. (1990) 11:116-28. doi: 10.1016/0197-2456(90)90005-m

18. Gu HF. Genetic and epigenetic studies in diabetic kidney disease. Front Genet. (2019) 10:507. doi: 10.3389/fgene.2019.00507

19. Han Q, Geng W, Zhang D, Cai G, Zhu H. ADIPOQ rs2241766 gene polymorphism and predisposition to diabetic kidney disease. J Diabetes Res. (2020) 2020:5158497. doi: 10.1155/2020/5158497

20. Fathy SA, Mohamed MR, Ali MAM, El-Helaly AE, Alattar AT. Influence of IL-6, IL-10, IFN-gamma and TNF-alpha genetic variants on susceptibility to diabetic kidney disease in type 2 diabetes mellitus patients. Biomarkers. (2019) 24:43-55. doi: 10.1080/1354750X.2018.1501761

21. Yu K, Zhang J, Zhang J, Dou C, Gu S, Xie Y, et al. Methionine synthase A2756G polymorphism and cancer risk: a meta-analysis. Eur J Hum Genet. (2010) 18:370-8. doi: 10.1038/ejhg.2009.131

22. Niu WQ, Zhang Y, Ji KD, Gao PJ, Zhu DL. Contribution of five top wholegenome association signals to hypertension in Han Chinese. J Hum Hypertens. (2011) 25:278-80. doi: 10.1038/jhh.2010.114

23. Stanton RC. Diabetic kidney disease and hypertension. Exp Clin Endocrinol Diabetes. (2016) 124:93-8. doi: 10.1055/s-0035-1559760

24. Weinrauch LA, Bayliss G, Segal AR, Liu J, Wisniewski E, D’Elia JA. Treating hypertension in diabetic patients with advanced chronic kidney disease: what should we have in mind? J Clin Hypertens (Greenwich). (2016) 18:1077-8. doi: $10.1111 /$ jch. 12839

25. Frossard PM, Obineche EN, Lestringant GG. Association of an apolipoprotein B gene marker with essential hypertension. Hypertension. (1999) 33:1052-6. doi: 10.1161/01.hyp.33.4.1052

26. Zhao WY, Huang JF, Wang LY, Li HF, Zhang PH, Zhao Q, et al. Association of the apolipoprotein $\mathrm{B}$ gene polymorphisms with essential hypertension in Northern Chinese Han population. Biomed Environ Sci. (2007) 20:260-4.

27. Yoshida T, Kato K, Yokoi K, Watanabe S, Metoki N, Satoh K, et al. Association of candidate gene polymorphisms with chronic kidney disease in Japanese individuals with hypertension. Hypertens Res. (2009) 32:411-8. doi: $10.1038 /$ hr.2009.22

Conflict of Interest: The authors declare that the research was conducted in the absence of any commercial or financial relationships that could be construed as a potential conflict of interest.

Copyright (c) $2021 \mathrm{Ma}$, Wang, Zhao, Yu, Deng, Jiang, Cao, Li and Niu. This is an open-access article distributed under the terms of the Creative Commons Attribution License (CC BY). The use, distribution or reproduction in other forums is permitted, provided the original author(s) and the copyright owner(s) are credited and that the original publication in this journal is cited, in accordance with accepted academic practice. No use, distribution or reproduction is permitted which does not comply with these terms. 